\title{
THE ANTIPODE OF A FINITE-DIMENSIONAL HOPF ALGEBRA OVER A FIELD HAS FINITE ORDER
}

\author{
BY DAVID E. RADFORD
}

Communicated by Barbara L. Osofsky, June 20, 1975

The purpose of this note is to indicate a proof of the fact that the order of the antipode of a finite-dimensional Hopf algebra over a field has finite order. It has been shown [2], [5] that the order of the antipode of an infinite-dimensional Hopf algebra is not necessarily finite; and the order of the antipode is finite in the finite-dimensional case if the Hopf algebra is unimodular [1] or pointed and the ground field has prime characteristic [6]. Using the bilinear form introduced and studied in [3] we prove that the order is finite for any finite-dimensional Hopf algebra over a field.

The bilinear form, integrals, grouplike elements, one-dimensional ideals, and the antipode are all related in rather intriguing ways. The proof of the finite order theorem is based on an explicit formula describing the fourth power of the antipode, as suggested by Theorem 5.5 of [1]. Full details will appear elsewhere [4].

1. The bilinear form. Let $A$ be a finite-dimensional Hopf algebra over a field $k$. Then the linear dual $A^{*}$ is also a Hopf algebra. An element $x \in A$ is called a left integral if $h x=\epsilon(h) x$ for all $h \in A$. In [6] it is shown that the space of left integrals is one dimensional, and if $0 \neq m \in A^{*}$ is a left integral then $A^{*}=$ $A \cdot m$ (hence $A^{*}$ is a free left $A$-module). Let $0 \neq m \in A^{*}$ be a left integral. Then the associative bilinear form $\beta: A \times A \rightarrow k$ defined by $\beta(a, b)=m(a b)$ is nonsingular.

The bilinear form $\beta$ lies at the foundation of the analysis of the antipode. Using it one can derive much information about bijective bialgebra maps as well (for example $t=s^{2}$ where $s$ is the antipode of $A$ ). If $t: A \rightarrow A$ is any bijective bialgebra map then one can compute directly that $\beta(t(a), b)=\beta\left(a, \omega t^{-1}(b)\right)$ for some $0 \neq \omega \in k$. Thus the transpose $t^{*}$ of $t$ with respect to the bilinear form $\beta$ has a particularly nice characterization. Using the fact that $t^{*}=\omega t^{-1}$ it follows that the eigenvalues $\lambda_{1}, \ldots, \lambda_{r}$ of $t$ are also $\lambda_{1}^{-1} \omega, \ldots, \lambda^{-1} \omega$ (in fact the relationship $\operatorname{dim} A_{\lambda}=\operatorname{dim} A_{\lambda-1} \omega$ holds for eigenspaces, $\lambda \neq 0$ ). The invariant factors of $t$ possess a high degree of symmetry dependent on $\omega$.

AMS (MOS) subject classifications (1970). Primary 16A50, 16A58, 16A60; Secondary 15 A25, 15 A 30.

Key words and phrases. Hopf algebra, order of antipode, bilinear form, bialgebra morphism. 
Now let $x \cdot m=\epsilon$. Then $x$ is a left integral. Let $\beta^{*}: A^{*} \times A^{*} \rightarrow k$ be the bilinear form corresponding to $x$. Let $\beta_{l}(a)=m \cdot a, \beta_{r}(a)=a \cdot m$, and let $\beta_{l}^{*}$ and $\beta_{r}^{*}$ be the analogues for $\beta^{*}$. Larson and Sweedler proved [3] that

$$
s=\beta_{r}^{*} \circ \beta_{l} \quad \text { where } s \text { is the antipode of } A .
$$

By generalizaing the notion of left integral and modifying (1.1) we are able to prove the main theorem of this paper.

2. Integrals. From $\S 1$ recall $A^{*}=A \cdot m$ where $m$ is a nonzero left integral. If $\eta \in \operatorname{Alg}(A, k)$ then $x \cdot m=\eta$ for some $x \in A$. Notice $h x \cdot m=h \cdot(x \cdot m)$ $=h \cdot \eta=\eta(h) x \cdot m$ so $h x=\eta(h) x$. Let $L_{\eta}=\{x \in A: h x=\eta(h) x$ all $h \in A\}$ be the space of (left) $\eta$-integrals. For $g \in G(A)=\operatorname{Alg}\left(A^{*}, k\right)$ we define the space of left $g$-integrals $L_{g}$ in the same manner. $R_{\eta}$ and $R_{g}$ are defined in the obvious way.

2.1. Proposition. Let $A$ be a finite-dimensional Hopf algebra over a field k. Then

(a) $\operatorname{Alg}(A, k) \rightarrow$ \{one-dimensional ideals of $A\}, \eta \mapsto L_{\eta}$, is bijective.

(b) There exists a unique $\alpha \in \operatorname{Alg}(A, k)$ such that $R_{\eta \alpha}=L_{\eta}=R_{\alpha \eta}$ for all $\eta \in \operatorname{Alg}(A, k)$.

Notice that $\alpha$ is in the center of $G\left(A^{*}\right)=\operatorname{Alg}(A, k)$, and that $A$ is unimodular if and only if some one-dimensional ideal of $A$ is in the center of $A$.

Let $a \in G(A)$ be the counterpart of $\alpha$ in 2.1 .

2.2. Proposition. Let $A$ be a finite-dimensional Hopf algebra with antipode s over a field $k$.

(a) $t^{*}=\alpha(a) t^{-1}$ for $t=s^{2}$ and $\beta$ as in $\S 1$.

(b) If $x \in L_{\eta}$ then $s(x)=\eta^{-1}(a) \alpha \eta^{2} \rightarrow x$. Thus if $x$ is a left integral then $s(x)=\alpha \rightarrow x$.

(c) $s\left(L_{\eta}\right)=L_{\eta}$ if and only if $\eta^{-2}=\alpha$.

One interesting consequence of $2.2(\mathrm{c})$ is that $G\left(A^{*}\right)$ has odd order if and only if $A$ has a unique one-dimensional ideal invariant under $s$.

3. The main theorem. Let $A, \beta, \beta^{*}, \alpha$, and $a$ be as in $\S \S 1$ and 2. Denote by $A^{\text {op }}$ the Hopf algebra obtained from $A$ by "twisting" multiplication (e.g., $h \cdot k$ $=k h ; h, k \in A)$, and by $A^{\text {cop }}$ the Hopf algebra obtained from $A$ by "twisting" comultiplication (e.g., $\delta(h)=\Sigma h_{(2)} \quad h_{(1)}$ for $h \in A$ ). By formulating (1.1) for the Hopf algebras $A^{\mathrm{op}}, A^{\mathrm{cop}}$ and $\left(A^{\mathrm{op}}\right)^{\mathrm{cop}}$ as well the following is proved:

\subsection{Lemma. Let $A$ be a finite-dimensional Hopf algebra with antipode $s$} over a field.

(a) $\beta_{r}\left(s^{2}(h)\right)=\beta_{l}(\alpha \rightarrow h)$ and

(b) $\beta_{l}\left(s^{2}(h)\right)=\beta_{r}\left(a^{-1}\left(h \leftarrow \alpha^{-1}\right) a\right)$ for $h \in A$. 
From the lemma one computes $\beta_{r}\left(s^{4}(h)\right)=\beta_{l}\left(\alpha \rightarrow s^{2}(h)\right)=\beta_{l}\left(s^{2}(\alpha \rightarrow h)\right)=$ $\beta_{r}\left(a^{-1}\left(\alpha \rightarrow h<\alpha^{-1}\right) a\right)$ so since $\beta$ is nonsingular,

$$
s^{4}(h)=a^{-1}\left(\alpha \rightarrow h-\alpha^{-1}\right) a \quad \text { for all } h \in A .
$$

Since grouplike elements are independent the formula implies:

3.3. THEOREM. The order of the antipode of a finite-dimensional Hopf algebra over a field is finite.

Sweedler has observed the theorem implies that the powers of $s$ are semisimple operators in characteristic 0 . Waterhouse [7] has recently shown that the theorem is true for finite Hopf algebras.

\section{REFERENCES}

1. R. G. Larson, Characters of Hopf algebras, J. Algebra 17 (1971), 352-368. MR 44 \#287.

2. - The order of the antipode of a Hopf algebra, Proc. Amer. Math. Soc. 21 (1969), 167-170. MR $39 \# 1524$.

3. R. G. Larson and M. E. Sweedler, An associative orthogonal bilinear form for Hopf algebras, Amer. J. Math. 91 (1969), 75-94. MR 39 \#1523.

4. D. E. Radford, The order of the antipode of a finite-dimensional Hopf algebra is finite, Amer. J. Math. (to appear).

5. M. E. Sweedler, Hopf algebras, Math. Lecture Note Series, Benjamin, New York, 1969. MR 40 \#5705.

6. E. J. Taft and R. L. Wilson, On antipodes in pointed Hopf algebras, J. Algebra 29 (1974), 27-32. MR 49 \#2820.

7. W. C. Waterhouse, Antipodes and grouplikes in finite Hopf algebras (to appear).

DEPARTMENT OF MATHEMATICS, LAWRENCE UNIVERSITY, APPLETON, WISCONSIN 54911

Current address: Department of Mathematics, Rutgers University, New Brunswick, New Jersey 08903 